\title{
UNASUR: alternativa de integración frente a desafíos internacionales emergentes
}

\author{
UNASUR: an alternative for integration in the face \\ of emerging international challenges
}

\author{
Paola Andrea Baroni* \\ María Florencia Rubiolo**
}

\section{Resumen}

El presente trabajo pretende analizar las razones por las cuales América del Sur necesita configurar un espacio regional de diálogo frente a los desafíos de un mundo crecientemente multipolar. En este sentido la Unión de Naciones Suramericanas (UNASUR) aparece como el proyecto regional con mayores posibilidades de convertirse en un foro a través del cual se pueda alcanzar consensos y establecer posturas regionales comunes. Concentraremos nuestra atención en el caso de China porque se presenta como una alternativa real a los Estados Unidos y a la Unión Europea en términos comerciales y de inversión. A su vez, el tamaño de su economía, permite pensar en la inserción de toda América del Sur, elemento que favorecería la cooperación.

Palabras clave: UnASUR, América del Sur, cooperación regional, integración, República Popular China

Universidad Empresarial Siglo 21, Córdoba, Argentina. <pbaroni@vesiglo21.edu.ar>.

** Centro de Estudios Avanzados, Unidad Ejecutora de CONICET (Consejo Nacional de Investigaciones Científicas y Técnicas), Argentina.<frubiolo@gmail.com>

Recibido el 27 de octubre de 2009. Aceptado el 16 de diciembre de 2009. 


\section{Abstract}

This paper aims to analyze the reasons why South America needs to establish a regional arena of dialogue in the face of challenges in an increasing multipolar world. We consider UNASUR as the regional initiative with greater possibilities of turning into a forum through which consensus can be reached and common regional stands established. We will focus on the case of the People's Republic of China because it appears as a real alternative to the United States and the European Union in the commercial and investment fields. At the same time, the size of its economy, allows considering the insertion of the whole South America, an element that would favor cooperation.

KEY WORDS: UNASUR, South America, regional cooperation, integration, People's Republic of China. 
Paola Baroni et al. • UNASUR: alternativa de integración frente a desafíos...

\section{INTRODUCCIÓN}

El sistema internacional contemporáneo presenta, a nuestro entender, un multipolarismo creciente. Es decir, aunque coincidimos con las visiones que afirman que en las áreas política y estratégica el poder continúa concentrado en un polo, observamos en el área económica el constante crecimiento de diversos centros a nivel mundial. Esta multiplicación favorece el dinamismo de los lazos comerciales y financieros $y$, al mismo tiempo, estimula la diversificación de las relaciones exteriores. A pesar de los beneficios que este escenario multipolar puede reportar a los Estados en términos comerciales y probablemente políticos consideramos que, como contraparti$\mathrm{da}$, la competencia se convierte en el rasgo cada vez más distintivo de las relaciones interestatales. El crecimiento de países como China e India ha llevado a una demanda creciente de recursos naturales cada vez más escasos. Si sumamos esto al incremento de población en países en vías de desarrollo podemos explicar la modificación del patrón del comercio internacional, donde los commodities y las materias primas incrementaron sustancialmente su valor.

Dentro de este dinámico y competitivo escenario internacional, el posicionamiento estatal y regional se convierte en una variable cada vez más determinante. Esta variable entra en juego tanto para enfrentar las amenazas y aprovechar las oportuni- dades que traen consigo el crecimiento y la expansión internacional de nuevos polos económicos, como para otorgar un mayor peso a la voz nacional o regional ante otros Estados. Una de las estrategias que los Estados adoptan para alcanzar este doble desafío es la integración regional. Es claro que los países deciden integrarse por el beneficio que dicha política generará a la sociedad toda. La interdependencia y la globalización han hecho de la integración una herramienta más que útil para que los Estados potencien sus recursos y capacidades y salven sus defectos y carencias.

Estos procesos se diferencian, sin embargo, por las metas que persiguen de acuerdo con las capacidades de los miembros que los integran. Las iniciativas de integración entre países del Sur presenta características propias (Olivet 2005: 22), entre ellas la principal motivación es la diversificación mientras que en los países del Norte es el balance de poder. En el primer caso, lo que se busca es superar el obstáculo que presenta la relación con pocos socios, evitar la exclusión de ciertas regiones y tener mayor participación en la economía mundial, evitando así quedar en la periferia. Dentro de este contexto, nuestro interés se centra en Sudamérica donde históricamente dichos procesos de integración no han tenido el éxito esperado. Creemos que la importancia dada a las cuestiones meramente comerciales, sin tener en cuenta los otros aspectos de la integración, llevaron a estos pseudo-fracasos 
y a la pérdida de posición internacional de la región. La competencia comercial, alimentada por la asimetría en la distribución de los recursos, combinada con la necesidad de insertarse en un sistema económico internacional cada vez más exigente, ha llevado a que la principal característica de las relaciones interregionales haya sido, en términos de Cesarín, el unilateralismo competitivo (Cesarín 2008:26). Estas prácticas han actuado en detrimento de la construcción de un espacio regional de concertación que se presenta como una condición sine qua non si la región pretende insertarse en el mundo con una voz más fuerte.

El presente trabajo pretende -entonces- analizar las razones por las cuales América del Sur necesita configurar un espacio regional de diálogo que le permita superar estas experiencias del pasado y coordinar políticas para enfrentar los desafíos, y aprovechar las oportunidades que presenta un mundo crecientemente multipolar. En este sentido la Unión de Naciones Suramericanas (UNASUR) aparece, a nuestro entender, como el proyecto regional con mayores posibilidades de convertirse en un foro a través del cual se pueda alcanzar consensos y establecer posturas regionales comunes ante los principales centros de poder externos.

Analizaremos la constitución de este espacio regional a través de los desafíos que conlleva la llegada de nuevos actores de peso internacional a América del Sur. Centraremos nuestra atención en el caso de China porque se presenta como una alternativa real a los Estados Unidos y a la Unión Europea en términos comerciales y de inversión. A su vez, el tamaño de su economía permite pensar en la inserción de toda América del Sur, elemento que favorecería la cooperación. El espacio regional de diálogo sería, entonces, un primer paso hacia la conformación de una región que se constituya en polo de atracción para China, ya que América del Sur no es un área prioritaria para la RPCh debido a la distancia geográfica y cultural. A pesar de ello, China busca -a través de una política exterior cada vez más asertiva- alcanzar una posición de prestigio y poder en la escena internacional, y es dentro de esta política global donde América del Sur puede ocupar un lugar de relevancia dada la complementariedad de las economías.

La estructura del trabajo constará de una presentación de los antecedentes de integración en América del Sur con la evaluación de sus avances y fracasos, y la introducción de las principales características de UNASUR en la actualidad. Continuaremos con el análisis de las circunstancias en las cuales nuevos Estados extrarregionales intentan ingresar en la región, distinguiendo el caso de China. Abordaremos tanto las características de su política exterior global como las particularidades de la misma hacia América Latina y sus intereses en la región, resaltando cómo estos pueden actuar en detrimento de la integración. Como complemento de este apartado, especificaremos y expli- 
Paola Baroni et al. • UNASUR: alternativa de integración frente a desafíos...

caremos las motivaciones de los países de America Latina en su relación con estos Estados, nuevamente centrándonos en el gigante asiático. En este punto procuraremos subrayar los obstáculos a superar en el proceso de constitución de la región en un polo de atracción que permita diversificar las relaciones exteriores. Por último expondremos las razones por las cuales creemos que UNASUR puede convertirse en el espacio de diálogo y consenso que América del Sur necesita para sobreponerse a los contratiempos que presentan estos Estados extrarregionales como paso previo para un mejor posicionamiento internacional.

\section{ANTECEDENTES Y \\ CARACTERÍSTICAS REGIONALES DE INTEGRACIÓN}

Los intentos de integración que se han abordado desde la región son numerosos pero en general no han sido exitosos. La Asociación Latinoamericana de Libre Comercio (ALALC) fue el primero de estos proyectos regionales. Se creó en 1960 con la intención de eliminar las barreras al comercio entre los Estados miembros. Sin embargo se encontró con obstáculos de política interna como la política de sustitución de importaciones de los países, que impidieron la consecución de los objetivos y propiciaron su disolución en 1980 (Pinheiro Guimaraes, 2007: 115). Su sucesor fue la Asociación Latinoamericana de Integración (ALADI) creada por el Tratado de Montevideo en 1980. Incluye actualmente a 13 países de Centroamérica y América del Sur -con la reciente incorporación de Panamá-y tiende a la reducción de aranceles dentro de la región con el objetivo mayor de conformar un mercado común latinoamericano. Las metas de integración se basan en criterios más flexibles, graduales y progresivos. Dada la heterogeneidad productiva y de estrategias de inserción comercial de sus miembros, el objetivo de conformar un mercado común parece más difícil de alcanzar. De todos modos, continúa generando un marco jurídico para los acuerdos comerciales entre los países de América Latina (García Moritán, 2007: 26).

Dos proyectos de integración de menor alcance geográfico, aunque con un espectro temático más amplio, son el Mercado Común del Sur (MERCosur) y la Comunidad Andina de Naciones (CAN), sucesora del Pacto Andino creado en 1969 por Chile, Perú, Ecuador, Bolivia, Colombia y Venezuela. Sus objetivos son la aceleración de la integración económica entre los miembros, la coordinación del desarrollo industrial regional, la regulación de la inversión extranjera y el mantenimiento de un arancel externo común ${ }^{1}$. El MERCOSUR fue creado por iniciativa de los presidentes de Argentina y Brasil en 1985 como un proceso de integración bilateral

1 Para más información ver: www.comunidadandina.org . 
al cual se sumaron Uruguay y Paraguay en 1991, dando origen formal al proceso regional. Surgió como un proceso enmarcado en las premisas del Consenso de Washington, considerando al libre comercio como instrumento suficiente para la promoción del desarrollo, la reducción de las desigualdades sociales y la generación de empleos (Pinheiro Guimaraes, 2007: 116). Estos lineamientos pueden observarse en algunos de sus objetivos anunciados en el Tratado de Asunción: «libre circulación de bienes, servicios y factores productivos entre los países; eliminación de los derechos aduaneros y restricciones no arancelarias y a circulación de mercaderías y cualquier otra medida equivalente; establecimiento de un arancel externo común y adopción de una política comercial común con relación a terceros estados...». ${ }^{2}$

A pesar del activismo que hubo en materia de integración, la realidad pone de manifiesto que ninguno de estos procesos alcanzó completamente sus objetivos. Este fenómeno puede atribuirse a una multiplicidad de factores. En primer lugar, observamos que a pesar de la complementariedad que existe entre las economías de la región y de los acuerdos bilaterales internacionales e interregionales existentes en esta materia, ha habido poca intensidad de la interdependencia económica. Esto se refleja en las exportaciones intrarregionales, las cuales han representado el $18,46 \%$ del total de las exportaciones de la región para el año 2006.

Cuadro 1

América del Sur en 2006: exportaciones por paises (en miles de dólares y porcentaje de total)

\begin{tabular}{|l|r|r|r|r|r|r|r|r|r|r|r|}
\hline & Argentina & Brasil & Venezuela & Chile & Bolivia & Colombia & Ecuador & Paraguay & Perú & Uruguay & $\begin{array}{c}\text { América } \\
\text { del Sur }\end{array}$ \\
\hline Argentina & 0 & 11171380 & 396300 & 768400 & 391200 & 51200 & 44200 & 168500 & 73700 & 301900 & \\
\hline Bolivia & 380500 & 693900 & 8200 & 285800 & 0 & 58500 & 9300 & 29500 & 184500 & 6100 & \\
\hline Brasil & 8140500 & 0 & 571100 & 2757800 & 1592000 & 190700 & 26500 & 328000 & 803600 & 584100 & \\
\hline Chile & 4403800 & 3896000 & 131400 & 0 & 68100 & 258800 & 554400 & 130800 & 1419400 & 166100 & \\
\hline Colombia & 550800 & 2128800 & 1166200 & 491500 & 155800 & 0 & 715200 & 2600 & 501100 & 15500 & \\
\hline Ecuador & 302400 & 873300 & 279500 & 422000 & 10900 & 1237100 & 0 & 3900 & 325700 & 8100 & \\
\hline Guyana & 2200 & 20100 & 24100 & 1100 & 0 & 5800 & 0 & 0 & 1900 & 0 & \\
\hline Paraguay & 622700 & 1230400 & 800 & 61600 & 28400 & 2400 & 900 & 0 & 600 & 58300 & \\
\hline Perú & 728900 & 1500800 & 523100 & 931500 & 248700 & 691600 & 1039300 & 27800 & 0 & 21500 & \\
\hline Uruguay & 1174800 & 1006100 & 3500 & 89300 & 1300 & 5600 & 3000 & 420200 & 8400 & 0 & \\
\hline Venezuela & 809500 & 3555000 & 0 & 492500 & 201000 & 2698700 & 318900 & 9600 & 410100 & 78400 & \\
\hline Surinam & 4300 & 31200 & 1400 & 1100 & 100 & 9200 & 200 & 0 & 900 & 200 & \\
\hline Total Am. Sur & 17120400 & 26106980 & 3105600 & 6302600 & 2697500 & 5209600 & 2711900 & 1120900 & 3729900 & 1240200 & 69345580 \\
\hline Total en \% & $36,78 \%$ & $18,99 \%$ & $4,72 \%$ & $11,27 \%$ & $63,87 \%$ & $21,37 \%$ & $21,30 \%$ & $58,80 \%$ & $16,40 \%$ & $31,11 \%$ & $18,46 \%$ \\
\hline Total CEPAL & 46545000 & 137466600 & 65692600 & 55880400 & 4223100 & 24374500 & 12727700 & 1906200 & 22736800 & 3985700 & 375538600 \\
\hline
\end{tabular}

Fuente: Anuario estadístico de la CEPAL 2008 y elaboración propia.

2 Ver Tratado de Asunción. 
Paola Baroni ET AL. • UNASUR: alternativa de integración frente a desafíos...

Una de las principales consecuencias de este bajo nivel de interdependencia comercial es que los incentivos para la integración son menores. En segundo lugar, las asimetrías de la región -tamaño de los países, coeficiente de apertura, diversificación de la estructura productiva, recursos energéticos, nivel de desarrollo político e ideológico- llevan a una distribución desigual de los incentivos para cooperar y generan una resistencia a asignar discrecionalidad (Bouzas, Da Motta, Rios, 2008: 323). En tercer lugar, todo proceso de integración necesita de un líder que guíe y coordine ante posibles divergencias en el proceso. En el caso de América del Sur, la existencia de un líder regional que además de cumplir estas funciones provea de bienes públicos regionales, incluso a expensas de sus intereses inmediatos, no es evidente. Según Bouzas, Da Motta y Ríos, en el caso del MERCOSUR, Brasil sería el proveedor natural pero ha tenido una oferta esporádica y reactiva (Bouzas et al, 2008: 327). En cuarto lugar, las prácticas unilaterales de los países de la región han minado los esfuerzos de integración. Estas políticas, denominadas por Cesarín como unilateralismo competitivo (Cesarín, 2008: 26), se materializan en las liberalizaciones comerciales unilaterales, incluso en países que estaban comprometidos en procesos multilaterales de integración comercial.

El principal efecto de estos elementos sobre la integración ha sido alimentar escepticismo respecto de los beneficios del avance de los procesos; a lo que se suma la continuación de una desconfianza histórica entre los Estados de América del Sur que contribuye a desalentar la profundización de los lazos. Se agrega a este condicionante histórico la falta de metodología para superar los anteriores obstáculos. Es decir, no hay una definición común de objetivos, métodos y prioridades en la región. De acuerdo a Bouzas y otros esto se debe a la distinta funcionalidad política que se adjudica al proceso de integración subregional (Bouzas, 2008: 340). Por lo tanto, los procesos de integración han llegado a tal punto de politización por parte de los Estados que los integran que han perdido el foco de atención. Un ejemplo de ello es la forma en que se ha intentado avanzar en la integración comercial. En muchos casos, la liberalización de los productos se ha dado en aquellos con aranceles de importación menores y se han mantenido protegidos bienes que tienen aranceles elevados porque muchos países siguen usando a las exportaciones e importaciones como fuente de ingresos y como barreras para proteger a industrias nacionales no competitivas internacionalmente.

A pesar de que las iniciativas de integración regional no reflejan, a nuestro parecer, avances significativos en los niveles de cooperación, ni en el logro de los objetivos planteados en la gestación de los procesos, creemos que la supervivencia de procesos subregionales demuestra la existencia de la práctica de la integración entre los países de 
América del Sur. Esto podría facilitar una integración regional más amplia, si consideramos que la persistencia de dichas interacciones ha permitido construir ciertos lazos y por ende, podría favorecer el reconocimiento de intereses comunes que superen las rivalidades. El Grupo Contadora, el Grupo de Apoyo a Contadora y el Grupo de Río, una derivación de los anteriores que toma forma en 1990, son algunos predecesores. El Grupo de Río, de más reciente formación, tiene como función principal ejercer una mediación colectiva para dirimir e intentar solucionar conflictos que involucren a países de la región (Peña, 2008). Completan este listado las Cumbres Iberoamericanas, la Organización de los Estados Americanos (OEA), la Comisión Económica para América Latina y el Caribe (CEPAL), el Sistema Económico Latinoamericano y del Caribe (SELA), la Iniciativa para la Integración de la Infraestructura Regional Sudamericana (IIRSA) y la Alternativa Bolivariana para América (ALBA). Este último grupo comprende iniciativas de mayor alcance geográfico, de diversidad temática y con grados de institucionalización variable.

El más reciente proyecto de integración regional sudamericano es UNASUR. En el año 2000, el gobierno de Brasil convocó a la primera Cumbre de Presidentes de países de América del Sur en Brasilia. En dicha reunión se incorporó en la agenda regional la cuestión de la infraestructura. Esto dio origen a IIRSA. El propósito de la misma ha sido movilizar recursos y cooperar para la construcción de ejes multinacionales de integración y desarrollo con el objeto de facilitar la comunicación y disminuir los costos de transacción dentro de la región (Bouzas y otros, 2008: 336-337). Esta Cumbre se convirtió en el primer paso hacia la constitución de la Comunidad Sudamericana de Naciones (CSAN) que recién tomaría forma en el 2004, en Cuzco. Los mandatarios se reunieron por primera vez, en el marco de esta iniciativa, en Brasilia en 2005. En este encuentro se estableció una agenda de prioridades económicas y políticas amplia que puso de manifiesto importantes divergencias en cuanto a los objetivos y métodos de la CSAN. Los dos puntos principales fueron: por un lado, fortalecer la institucionalidad existente respecto de la integración regional (ALADI, CAN y MERCOSUR), y por otro, la convergencia de estos procesos en un área de libre comercio sudamericana. La comisión designada para trabajar sobre estos objetivos, sin embargo, no respetó el espíritu originario de los mismos. Propuso el fortalecimiento de la arquitectura institucional de la CSAN y la búsqueda de una integración económica y productiva más amplia que la presentada en Brasilia, que debía ser complementada por formas de cooperación política, social y cultural (Comisión Estratégica de Reflexión, 2006). De esta manera, no solo se quitó el énfasis a los temas comerciales, sino también se restó prioridad a los procesos de integración existentes.

En la Declaración de Cochabamba (2006), los jefes de Estado se hicieron 
Paola Baroni et al. • UNASUR: alternativa de integración frente a desafíos...

eco de muchas de las recomendaciones de la Comisión de Reflexión. Uno de ellos fue la identificación de los campos de cooperación a través de la creación de grupos de trabajo dedicados a los temas de energía, infraestructura y política social. Al mismo tiempo se relegaron los temas comerciales, también siguiendo las indicaciones propuestas por la Comisión. Estas medidas, que postergan la tradicional prioridad de los procesos de integración sudamericano -los temas comerciales-, parecen reflejar la inquietud de los mandatarios por abordar lo que consideran los principales obstáculos al avance de la integración subregional: las asimetrías estructurales, la dimensión productiva y de desarrollo (Bouzas y otros, 2008: 339). Sin embargo, no siguieron las propuestas sobre la institucionalización de la Comunidad. En este sentido, se optó por la constitución de una Comisión de Funcionarios de Alto Nivel y una Secretaría pro témpore.

La CSAN fue reemplazada por la Unión de Naciones Suramericanas en abril de 2007, en la primera Cumbre energética sudamericana realizada en Venezuela. Los fines y principal objetivo de la misma, de acuerdo a la organización, son:

La Unión de Naciones Suramericanas busca el desarrollo de un espacio integrado en lo político, social, cultural, económico, financiero, ambiental y en la infraestructura. Este nuevo modelo de integración incluirá todos los logros y lo avanzado por los procesos del Mercosur y la Comunidad Andi- na, así como la experiencia de Chile, Guyana y Suriname. El objetivo último es y será favorecer un desarrollo más equitativo, armónico e integral de América del Sur. ${ }^{3}$

Las áreas prioritarias que ha establecido la organización son: el diálogo político; la integración física; el medio ambiente; la integración energética; los mecanismos financieros sudamericanos; las asimetrías; la promoción de la cohesión social, de la inclusión social y de la justicia social; y las telecomunicaciones.

El liderazgo de Brasil parece plasmarse claramente en UNASUR. Es el único actor que conserva el interés en el proyecto tanto en su vertiente política como comercial (Bouzas y otros, 2008: 340-341). Sin embargo, ante las dificultades que la iniciativa ha despertado entre los países miembros, Brasil ha ejercido una menor presión en el avance del proceso de integración. Siguiendo esta misma línea, Peña afirma que UNASUR representa una política de liderazgo de Brasil que consiguió el consenso de los demás países. Al mismo tiempo, sostiene que el proceso de integración en América del Sur se vio favorecido por el impulso que Brasil le imprimió como respaldo a una estrategia de desarrollo orientada a la inserción internacional (Peña, 2008: 77-79).

Hasta aquí hemos revisado brevemente los antecedentes de integración

3 Para más información ver: http://www. comunidadandina.org/sudamerica.htm 
en América del Sur en términos institucionales, evaluando sus dificultades y considerando sus avances relacionados principalmente con la socialización que las prácticas han generado. Veremos en la próxima sección que, como consecuencia de la redefinición actual del sistema interestatal, la región debe enfrentar los desafíos que presenta el intento de inserción de nuevos actores económicos en la región. Este fenómeno tiene la potencialidad de convertirse en una amenaza a la integración de la región o, por el contrario, puede estimular la concertación de políticas y la construcción de consensos regionales orientados a lograr una inserción internacional de la región de forma colectiva.

\section{LA REgIÓN DE AMÉRICA DEL Sur COMO ESCENARIO DE INSERCIÓN DE NUEVOS ACTORES ECONÓMICOS INTERNACIONALES}

En este apartado centraremos nuestra atención en el rol de la República Popular China (RPCh) en América del Sur como nuevo actor de relevancia internacional. Lo tomaremos, sin embargo, como caso paradigmático y precursor en la búsqueda de inserción en esta región, aunque no único: India y Rusia también comienzan a aparecer en el escenario regional.

La política exterior de la RPCh, que entre 1949 y 1978 mostró una fuerte impronta ideológica, actualmente refleja el pragmatismo que caracteriza a las decisiones económicas y políticas de Pekín. Es decir, con la llegada de Deng Xiaoping se comienza a abandonar el elemento ideológico como condicionante de los lazos externos y se inicia un proceso de apertura internacional a través de la política de «Puertas Abiertas». Este proceso comienza de forma paralela al período de modernización económica de China. Es menester entender entonces que su política exterior en los últimos 20 años, desde el fin de la Guerra Fría, está orientada a mantener un ambiente internacional, y principalmente regional, estable que le permita concentrarse en el desarrollo económico interno. Esta es la primera característica general de la política exterior china.

El multipolarismo se presenta como el segundo factor característico. Su postura al respecto parte de la convicción de que el mundo está encaminado en un proceso que lleva inevitablemente a la confección de un mundo multipolar, dado que frente a las múltiples diferencias culturales, religiosas, étnicas, económicas y políticas es difícil pensar que un solo país pueda guiarlo por sí solo. Este proceso, afirma la dirigencia de la RPCh, además de estar en consonancia con las aspiraciones y los intereses de la mayoría de los países puede conducir a un mundo más pacífico y seguro (Ministerio de Relaciones Exteriores de la República Popular China, 2003). Esta reestructuración en la distribución de poder que comienza con el fin de la Guerra Fría, guiará, entonces, a la conformación 
Paola Baroni et al. • UNASUR: alternativa de integración frente a desafíos...

de un sistema multipolar en el cual la RPCh deberá tener un lugar en el grupo de las grandes potencias a través de su Ascenso Pacífico (Cheng, 1999: 178).

Un tercer elemento clave en su diseño es la importancia otorgada a las relaciones Sur-Sur. En el período inmediatamente posterior al inicio de las Cuatro Modernizaciones de 1978, las relaciones exteriores se orientaron a atraer inversores de países desarrollados con el objetivo de financiar el desarrollo propio. Hasta fines de los años ochenta se priorizaron las relaciones con las grandes potencias occidentales para facilitar la inserción internacional del país, mientras que los lazos con los países del hemisferio Sur se relegaron y con frecuencia derivaron en relaciones inestables o interrumpidas. Sin embargo, en 1989 la crisis de Tiananmen revirtió los avances en el proceso de integración internacional. Los países occidentales condenaron súbita y abiertamente la represión llevada a cabo. Las fuertes sanciones impuestas a China, conjugadas con el hundimiento del bloque soviético y de los países de Europa Oriental, sumieron al país en el aislamiento (Cheng, 1999: 177).

Con la intención de superar el aislamiento internacional, el gobierno reconoció la necesidad de revitalizar lazos con países del mundo en desarrollo, los cuales se mantuvieron en silencio después de los hechos. Esta orientación se mantiene en la actual política exterior del país, de acuerdo con la cual se reconoce como miembro de los países en desarrollo y manifiesta su intención de cooperar en el desarrollo de estos y, a diferencia de las potencias occidentales, a facilitar asistencia sin ningún tipo de condicionamiento (Ministerio de Relaciones Exteriores de la República Popular China, 2003). Este elemento nos lleva a la cuarta característica de la política exterior china, la ausencia de condicionalidades de cualquier naturaleza, consecuencia de su respeto por el principio de no injerencia en los asuntos internos, a excepción del no reconocimiento de Taiwán como gobierno legítimo de China.

Dentro de este diseño de política exterior, la región de América del Sur aparece como parte integrante aunque no clave. Es decir, la región se integra en la estrategia china de Ascenso Pacífico principalmente como proveedora de materias primas, productos básicos y energía. El país necesita de estos recursos para sostener el crecimiento y la modernización de su economía que le permitan una diferente inserción en el sistema económico internacional. Este ascenso ha sido impulsado, según el gobierno chino, por el capital, la tecnología y los recursos adquiridos por medios no violentos. De esta forma, quieren diferenciarse de las prácticas imperialistas que caracterizaron a las potencias del hemisferio occidental en el siglo XIX. Esta doctrina, que a partir del 2004 se comenzó a conocer como Doctrina del Desarrollo Pacífico, enfatiza la importancia del poder blando. Por la naturaleza intrínseca de 
este tipo de poder China necesita ejercer una activa diplomacia que permita su despliegue. La principal área de este ejercicio diplomático es el sudeste asiático. El país se acerca a esta región a través, principalmente, del multilateralismo como medio para sosegar la percepción de amenaza que su rápido crecimiento económico puede generar en los países y para favorecer la creación de lazos que le permitan construir un área sólida de influencia y apoyo en los foros internacionales. Sin embargo, se reserva la utilización de prácticas bilaterales para las cuestiones que considera más sensibles, como las estratégicas. Un ejemplo de ello es su negativa a resolver el problema de las islas del Mar Meridional de China a través de negociaciones multilaterales.

Por el contrario, en América Latina su estrategia de acercamiento es más variada, aunque aún manteniéndose dentro del ejercicio del poder blando.

En una primera instancia, la forma de aproximación hacia Sudamérica puede encuadrarse en la cooperación Sur-Sur como la definimos en párrafos anteriores. El apoyo que la RPCh ha brindado a los países de la región en las reuniones del G-20 es muestra de esta postura a nivel internacional. Los recursos que ha utilizado para insertarse en la región han sido: 1) participación en la institucionalidad hemisférica -status de observador permanente en la OEA y el Grupo de Río, participación en el Banco Interamericano de Desarrollo (BID), presencia de tropas chinas en Haití; 2) fomento del inter- regionalismo hacia América Latina y el Caribe -Foro de Cooperación América Latina Asia del Este (FOCALAE); 3) inserción en esquemas subregionales de cooperación e integración (MERCOSUR, CAN) y 4) alianzas bilaterales (Cesarín, 2008: 13).

Podemos ver que en lo referente a cuestiones de naturaleza política el gobierno chino adopta políticas multilaterales, mientras que en los temas que afectan áreas que el país considera estratégicas en su relación con América Latina -recursos naturales y energéticos- se acerca de forma bilateral. Al mismo tiempo, las características de la integración regional, principalmente la carencia de una estructura comercial institucionalizada y la escasa interdependencia económica, han impedido que China se aproxime a la región en su conjunto. Entonces, son dos las variables que observamos como condicionantes del diseño de política exterior de la RPCh hacia América Latina, uno de origen interno la sensibilidad de los temas estratégicos -y otro de origen regional, la falta de institucionalidad y la débil integración comercial.

Uno de los objetivos centrales de la política exterior china en el período post Tiananmen ha sido lograr el status de economía de mercado, por los beneficios que este status le reporta a las economías nacionales. En América del Sur ha intentado obtener reconocimiento a través de acuerdos bilaterales. Los países de la región que reconocen a su contraparte asiático como 
Paola Baroni ET AL. • UNASUR: alternativa de integración frente a desafíos...

economía de mercado son Venezuela, Chile, Brasil, Argentina y Perú (Embajada de la República Popular China en India, 2004).

En el esfuerzo por incrementar los niveles de intercambio comercial, se han abordado diferentes estrategias tanto desde los países de Sudamérica como del país oriental. Desde una perspectiva bilateral, los dos principales acuerdos de tinte comercial firmados por países de la región con la RPCh son los Acuerdos de Libre Comercio con Chile y con Perú. El primero de ellos firmado en 2005 y el segundo en abril de 2009 (China Daily, 2009). Venezuela también sobresale por la proliferación de acuerdos y tratados bilaterales firmados con China desde 2006. Los mismos son de variada naturaleza: política, energética, tecnológica, agrícola, de infraestructura, cultural, financiera, comunicacional, social y educativa, minera, y turística (Dirección general de prensa presidencial, 2006).

En términos de flujos comerciales, en 2007 el principal socio de China en América del Sur fue Brasil, con un total de 29.714 millones de dólares y Chile ocupó un lejano segundo lugar con 14.696 millones de dólares (National Bureau of Statistics of China, 2008). El incremento del intercambio entre China y América del Sur ${ }^{4}$ en general fue

4 En esta medición se incluyen: Argentina, Bolivia, Brasil, Chile, Colombia, Ecuador, Paraguay, Perú, Uruguay y Venezuela. No se incluyen: Suriname, Guyana, ni Guayana Francesa. significativo entre 2002 y 2007, dado que aumentó de un total de 10.721 millones de dólares a 72.220 millones de dólares (National Bureau of Statistics of China, 2008). Sin embargo, a pesar del aumento en términos absolutos, las diferencias que se perciben al interior de la región en cuanto al volumen del flujo comercial para cada país indican que las asimetrías existentes pueden profundizarse si se continúan practicando políticas bilaterales de cooperación con la RPCh (ver gráfico en la página siguiente).

Las inversiones que China realiza en la región también han cobrado mayor importancia en los últimos años. Han tomado la forma de inversión extranjera directa para la explotación y obtención de recursos naturales y materias primas. Ejemplo de ello es la formación de una empresa conjunta -joint venture- entre PetroChina y Petróleos de Venezuela (PDVSA) para la exploración y desarrollo de petróleo. En la misma, PetroChina participaría con un $40 \%$ del paquete accionario y Venezuela con un $60 \%$. Al mismo tiempo se conformará una empresa conjunta de transporte de petróleo y dos refinerías (China View, 2009). En Perú se realizaron inversiones en la producción de aluminio por parte de Chinalco y en Argentina se produjo la compra de la productora mineral de hierro por parte de MCC. Estos acuerdos permiten reconocer el interés estratégico que en términos energéticos China tiene en la región y la intención de ambas partes de favorecer el mutuo 
Gráfico 1:

Intercambio comercial China-América del Sur 2002-2007 en USD 1000

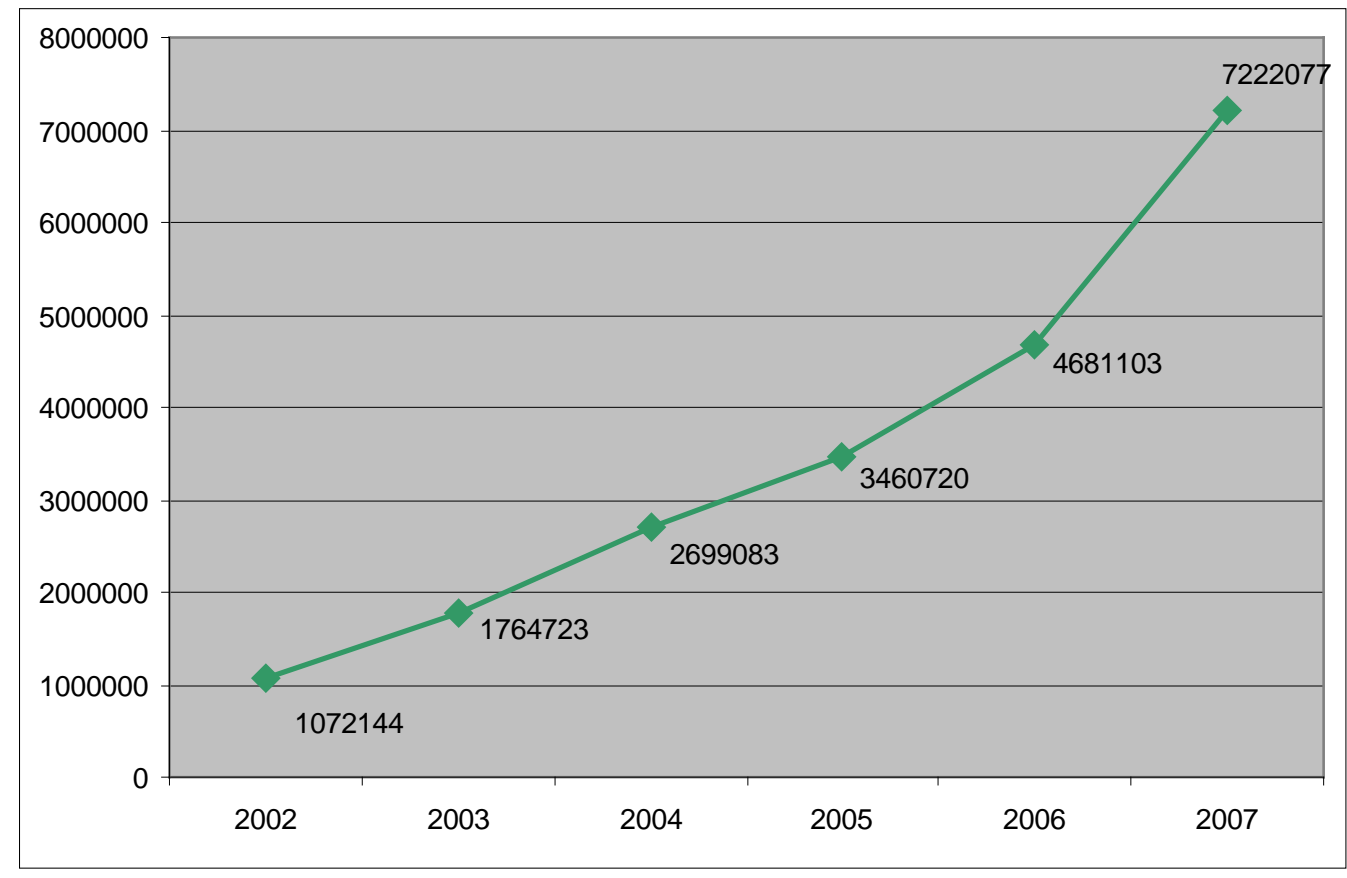

Fuente: National Bureau of Statistics of China, Yearbooks 2003 - 2008 y elaboración propia.

desarrollo a través del comercio internacional por medio de la cooperación.

A nuestro entender, China ingresa en América del Sur de forma despareja. Es decir, las diferencias entre las balanzas comerciales de los países sudamericanos respecto de la RPCh y el desequilibrio de los flujos de IED y conformación de joint ventures reflejan, en el corto plazo, una profundización de las asimetrías existentes en la región. Este proceso se alimenta con la persistencia de prácticas bilaterales que, paralelamente, desincentivan el avance de la integración. Es entonces que América del Sur, en este sistema económico crecientemente multipolar, enfrenta la necesidad de relacionarse con nuevos actores como China, India y Rusia, sin que esto deteriore los procesos de integración ni profundice las asimetrías regionales. América del Sur debe aprovechar la renovada importancia que el acercamiento de estos países le ha dado a la región para encontrar un rol de mayor relevancia en el sistema económico internacional. Enfrentar estos desafíos externos -que presentan amenazas de fragmentación, competencia y distribución desigual de beneficios- requiere de la coordinación de políticas nacionales a través de la construcción de consensos en torno 
Paola Baroni et al. • UNASUR: alternativa de integración frente a desafíos...

a las decisiones y acciones y también definiciones e interpretaciones regionales. Esto puede permitirle a la región convertirse en un polo de atracción, el principal desafío interno que se le presenta ante los cambios en la estructura económica internacional, a través de la diversificación de la producción y de los socios y la confección de un ámbito jurídico de seguridad.

\section{LOS DESAFÍOS INTERNOS DE AMÉrica del Sur ANTE LA LLEGADA DE NUEVOS ACTORES}

América del Sur en su relación con China intenta responder a dos principales intereses regionales: por un lado la necesidad de fortalecer tanto sus economías internas como sus instituciones nacionales y regionales; por otro, la de encontrar alternativas de inserción internacional respecto de Estados Unidos y la Unión Europea. En el primer sentido, creemos que la diversificación de productos y socios comerciales permitiría fortalecer las economías, y la segunda condición, permitiría ofrecer un marco de seguridad jurídica necesaria para la atracción de socios internacionales y capitales extranjeros. Para la consecución de ambos intereses la región necesita convertirse en un polo de atracción para países emergentes. En un plazo mayor, el fortalecimiento de las relaciones con nuevos Estados le permitirá alcanzar un mejor posicionamiento a nivel internacional.
Una de las necesidades de la región ha sido la diversificación de mercados que le permita actuar más libremente y no depender de pocos socios que condicionen su posición comercial. También es necesaria la diversificación de producción económica. La región depende altamente de la demanda de materias primas, recursos naturales y manufacturas básicas -sin valor agregado importante-, productos cuyos precios son sensibles ya que se ven fuertemente afectados por los vaivenes de la oferta y la demanda. China es un formador de precios de dichos productos por lo cual puede modificarlos al modificar su demanda. La bonanza que ha experimentado la región en este tema lleva al peligro de que se afiance el subdesarrollo estructural (Cesarín, 2008: 15) a menos que haya políticas nacionales y regionales de desarrollo que permitan diversificar la producción hacia nuevos mercados como China, ya que dicho país se ha convertido en el principal socio comercial de la mayoría de los países de la región, desplazando a Estados Unidos y la Unión Europea.

Esto también genera preocupación respecto de las importaciones desde China, que se basan en manufacturas baratas que están reemplazando en algunos países -como México y América Central- a los bienes de producción nacional. Por ello, reforzamos la idea de que hay que desarrollar Estados eficientes y competitivos pero que a la vez estén institucionalmente preparados para proteger su industria y po- 
sicionar sus productos (Roett, 2008: 286).

Las IED se han constituido en otra herramienta para el desarrollo, por lo tanto es importante que los Estados atraigan capitales extranjeros. Las empresas regionales ya están invirtiendo fuertemente dentro de América del Sur y es claro que China también tiene planes y proyectos concretos al respecto. Pero un problema que se plantea es la falta de seguridad jurídica en este aspecto: no hay protección extraterritorial para las inversiones por lo que la inestabilidad en esta área genera reticencias a la hora de invertir -sobre todo si tenemos en cuenta que la mayoría de ellas se dan en sectores sensibles como energía y servicios-. Claros ejemplos de ello han sido las nacionalizaciones llevadas a cabo por Bolivia, Argentina y Venezuela.

Entre los principales obstáculos que se presentan para la concertación de políticas encontramos la diversidad de orientaciones político-ideológicas que actúan en detrimento de la constitución y consolidación de instituciones regionales (Soares de Lima, 2008: 90). Esta variable contribuye a generar distintos modelos de integración dentro de la región, lo que dificulta el consenso en torno a políticas macro de cooperación regional. Un segundo elemento que se presenta como impedimento a la cooperación y que contribuye al deterioro de las instituciones regionales existentes, es la fragilidad de las políticas de integración del Estado. Es decir, los gobernantes privile- gian la atención de las demandas de su base electoral -consecuencia de la movilización y politización creciente de la sociedad civil-, sin tener en cuenta las repercusiones que este comportamiento puede tener sobre la integración regional (Soares de Lima, 2008: 105). Podemos sumar a estas condicionantes del nacionalismo en materia de recursos naturales, que resalta las tensiones regionales consecuencia de la asimetría estructural en su distribución. Esta característica condiciona la inserción internacional de los países de la región haciéndola más favorable para algunos.

En cuanto a las relaciones tradicionales de América del Sur con Estados Unidos y la Unión Europea, actualmente la región no es considerada un área de interés prioritario. De acuerdo a Tokatlián y Russell «hoy el peso específico de América Latina en la mayoría de los asuntos mundiales es menor que al término de la Segunda Guerra Mundial» (Russell, Tokatlián, 2008: 215). Para la UE, América Latina pierde prioridad frente a los desafíos internos que la región debe enfrentar relacionados con la ampliación de sus miembros y la dificultad que este proceso genera, frente a su relación con Estados Unidos en términos políticos y estratégicos, por el creciente desafío económico que presentan los países asiáticos, principalmente China (Von Grabendorff, 2008: 240). Este fenómeno se replica desde la perspectiva estadounidense, para la cual América Latina no es ni será una prioridad 
Paola Baroni et al. • UNASUR: alternativa de integración frente a desafíos...

dada la inexistencia de conflictos que amenacen directamente la seguridad política, en comparación con otras regiones del mundo. La pérdida de importancia estratégica de América del Sur en la agenda externa de Estados Unidos se complementa con una creciente resistencia a las preferencias estadounidenses, que se acentúa a partir de finales de la década de 1990. Consecuentemente Estados Unidos encuentra mayores dificultades para imponer su agenda económica y de seguridad (Von Grabendorff, 2008: 217).

Sin embargo, en América Latina puede observarse una clara división entre el Norte y el Sur, que puede medirse por el nivel de oportunidades y atracción que brinda Estados Unidos a los productos, servicios y habitantes de la región. Debido a esto, México y países de América Central y el Caribe se orientan cada vez más hacia Estados Unidos y Canadá. Estos países ganan a su vez influencia económica y política interna en Estados Unidos, consecuencia de los flujos migratorios. Después de los ataques del 2001 en Estados Unidos surge una sensación de vulnerabilidad en materia económica y de seguridad que favorece la construcción de una comunidad de América del Norte bajo el liderazgo estadounidense (Von Grabendorff, 2008: 240-241).

En América del Sur, como región, la marginalización dentro de la política exterior de Estados Unidos es mayor, lo que no significa que en términos bilaterales no exista una política exte- rior activa, principalmente con países que presentan temas de importancia estratégica. Esto actúa en detrimento de la visión de América del Sur como una región desde la óptica de Estados Unidos, porque se implementa una política exterior basada en una perspectiva que articula países y temas (Russell, Tokatlián, 2008: 214-215).

El lugar de privilegio relativo dado a América del Norte, sumado a la marginalización dentro de la agenda exterior europea, genera en América del Sur la necesidad de encontrar una alternativa que respalde su inserción internacional a partir de un mejor posicionamiento relativo en un mundo altamente competitivo. Desde un enfoque interno surge, entonces, a partir del 2004 el concepto de una comunidad sudamericana de naciones, actualmente UNASUR, y desde un enfoque externo la necesidad de profundizar y extender lazos con Estados emergentes que se conviertan en alternativas frente a Estados Unidos.

\section{La Unión Suramericana de Naciones COMO ESPACIO DE DIÁlOGO Y CONSENSO REGIONAL}

Hasta aquí hemos intentado dar cuenta de dos de los principales fenómenos que tienen un efecto directo en la integración de América del Sur. Por un lado, la llegada de actores externos, particularmente China, que ante la ausencia de políticas conjuntas viene a profundizar las asimetrías y los quie- 
bres regionales. Esto se produce por la búsqueda unilateral de los países de la región de encontrar, en la asociación con la contraparte asiática, el mayor beneficio posible en el corto plazo aunque esto implique renunciar a compromisos regionales e incluso quebrantarlos. Nuestra tesis sostiene que para evitar que el ingreso de nuevos actores en el escenario sudamericano repercuta negativamente en la integración, es necesario alcanzar consensos previos en torno a algunas definiciones, acciones y decisiones dentro de un espacio de diálogo regional. Este espacio, por otro lado, también puede permitir a los países abordar el segundo desafío que se les hace ineludible ante la reconfiguración del sistema económico internacional: convertirse en un polo de atracción a nuevos actores a través del fortalecimiento de las instituciones tanto nacionales como regionales y de la diversificación de su producción. Sostenemos que la iniciativa regional que mayores posibilidades tiene de convertirse en este espacio, debido a sus características, es UNASUR.

Este proceso, a diferencia de otras iniciativas, tiene -como mencionamos en párrafos anteriores- un tinte claramente político, un bajo grado de institucionalización, la presencia de un Estado -Brasil- que intenta motorizar la integración y la participación de todos los países de América del Sur, sin Estados Unidos.

Coincidimos con Félix Peña en la afirmación de que se presentan dos elementos claves para la construcción de un espacio sudamericano de integración: la conciliación del mismo con las múltiples redes a la que los países de la región también se integran y la construcción de instituciones que gocen de credibilidad (Peña, 2008: 79). Considerando el segundo de estos elementos, creemos que la institución de prácticas informales es una de las condiciones que facilitaría la posterior creación de instituciones confiables y funcionales. Observamos que en varios de los procesos anteriores emprendidos en la región se dio una importancia fundamental a la institucionalización pero que una vez establecidos los órganos formales, su funcionalidad no resultó ser la esperada o no se recurrió a ellos ante problemas entre los miembros. Al mismo tiempo, el establecimiento de compromisos formales como la sujeción a una tarifa externa común por parte de los miembros del MERCOSUR, aunque brinda un marco para las relaciones externas ante momentos de crisis internas -como las de 1999 y 2001 en Brasil y Argentina respectivamente- fue vista como una limitante a la recuperación económica. ${ }^{5}$ El establecimiento de prácticas de cooperación, que favorezcan el conocimiento mutuo y permitan

\footnotetext{
Un ejemplo de ello fue la decisión del Ministro de Economía argentino Domingo Cavallo en 2001 de aumentar las tarifas a la importación de bienes de consumo provenientes de mercados fuera del MERCOSUR y disminuirlas a la importación de capitales del mismo origen, con la intención de estimular la inversión (Gómez Mera, 2005: 121).
} 
Paola Baroni et al. • UNASUR: alternativa de integración frente a desafíos...

la discusión sobre definiciones de problemas comunes y planes de acción, es un paso previo a la construcción de instituciones. Es decir, la presencia de reglas laxas de cooperación permiten una mayor flexibilización en dos niveles. Desde un punto de vista interno, posibilitaría que países con asimetrías estructurales puedan insertarse en un mismo esquema de integración sin que ello perjudique o limite su desarrollo. ${ }^{6}$ Desde un punto de vista externo, podría conciliarse la integración regional con las múltiples redes de integración a las que los países pertenecen. De esta forma, con el paso del tiempo, podrían construirse instituciones confiables que resulten funcionales a los objetivos de la región.

En segundo lugar encontramos el liderazgo de Brasil como factor determinante en el establecimiento de prácticas de cooperación e integración regional. Brasil es un país de características únicas en América Latina, con aspiración de liderazgo regional y proyección extra hemisférica. En este sentido, desde el gobierno de Lula Da Silva Brasil ha puesto énfasis en el plano político sin desestimar la integración económica-comercial. Según Soares de Lima «...el principal instrumento que Brasil puede ofrecer es la coordinación de la acción colectiva de los países sudamericanos en las arenas multilaterales globales y regionales» tratan-

6 Uruguay fue un ejemplo de este fenómeno al manifestar su intención de retirarse del MERCOSUR para poder firmar un Tratado de Libre Comercio con Estados Unidos. do de construir una capacidad de influencia regional en las negociaciones internacionales. Además señala que puede «...ofrecer sus buenos oficios en la mediación de eventuales situaciones de conflicto dentro y entre los países sudamericanos», y así desalentar posibles acciones unilaterales de Estados Unidos (Soares de Lima, 2007: 181).

Este rol de líder tiene diversas implicancias: en un primer lugar, le exige a Brasil distanciarse de Estados Unidos para ser reconocido como líder por los países de la región, pero al mismo tiempo acercársele para facilitarle el ascenso internacional (Russell, Tokatlián, 2008: 223). En segundo lugar, es necesario que los países sudamericanos reconozcan que Brasil es líder en la región. Para que esto ocurra, Brasil, debe por un lado funcionar como motor de la integración en su papel de liderazgo y por el otro, asegurar la persecución no solo de sus intereses nacionales sino los de la región en su conjunto, como incentivo a la integración. Por último, los países de la región deben encontrar la forma de aprovechar el poder relativo de Brasil para perseguir intereses sudamericanos, pero también deben contribuir a la definición de las metas regionales de forma que no sean un mero reflejo de los intereses nacionales brasileros.

Entendemos que Brasil ha puesto en práctica una activa política exterior orientada a favorecer la integración regional, aunque no ha sido el único. La política exterior de Chávez ha buscado ser actor clave del reordena- 
miento político regional considerando a la integración del sur como elemento esencial para derribar a la globalización capitalista (Sanjuán, 2008:146). A través de su política exterior energética intenta influir a nivel regional y para lograrlo ha trasladado sus intereses hacia América del Sur, sin relegar su tradicional nexo con el Caribe. El esquema de integración que plantea Venezuela contiene numerosos elementos ideológicos: un discurso fuertemente latinoamericanista, la ausencia de jerarquías y la compensación de desigualdades a través de acuerdos políticos. Sin embargo consideramos que a partir de la excesiva retórica nacionalista, que ha despertado resquemores en la región, Venezuela ha perdido la posibilidad de proyectarse como centro de poder: «La excesiva retórica nacionalista no condujo a la convergencia regional en torno de una matriz común de pensamiento sudamericana, puesto que ni siquiera hay consenso acerca de los problemas básicos de la región» (Sanjuán, 2008: 173).

Se suma a esta característica que su agenda regional no coincide con las de los demás países del área, dado que estos, en su mayoría, buscan una inserción pragmática en un mundo crecientemente multipolar.

La existencia de un desafío externo común a la región (Choi, Lee, 2002) podría facilitar la consolidación de UNASUR como espacio de integración sudamericano destinado en primera instancia a encontrar formas comunes de enfrentar el ingreso de nuevos acto- res y, en una segunda etapa, a posicionar a América del Sur dentro del sistema económico internacional. En la actualidad los únicos países que parecen presentar una estrategia global de relación con la RPCh en América del Sur son Chile y Brasil. El resto presenta respuestas cuasi reaccionarias frente a las propuestas e intereses chinos. Este escenario mantendría la actual dinámica de las relaciones América del Sur-China que se caracterizan por ser dictadas por los intereses de este último (Cornejo, 2005: 24).

\section{Conclusión}

En la era de la globalización es claro que la inserción internacional es clave para el crecimiento y el desarrollo económico de las naciones. Sin embargo, para alcanzar este objetivo, los países sudamericanos deben operar como un conjunto político cohesionado. Esto implica la superación de las diferencias internas generadas por la persecución de intereses nacionales sin tener en cuenta las consecuencias que éstos generan al resto de la región. Es decir, parecen estar más interesados en la influencia regional de sus propios países que en la elaboración e implementación de reglas para el bien común de la región, elemento necesario para el éxito de cualquier proceso de integración.

A pesar de las experiencias de integración es evidente la falta de una cultura de cooperación interregional que resulte viable para lograr un diálogo 
Paola Baroni et al. • UNASUR: alternativa de integración frente a desafíos...

más efectivo (Von Grabendorff, 2008: 243). No se logra desarrollar una formulación de intereses comunes como región debido a la vigencia de un concepto exagerado de soberanía que no permite hacer concesiones al vecino. El provincialismo que afecta muchas de las confrontaciones entre los países latinoamericanos contribuye a minar el papel internacional de la región. Por lo tanto, falta un concepto compartido de política exterior de América Latina hacia las contrapartes, que considere los costos y beneficios de este tipo de relación. Alcanzar un peso regional considerable frente a otros actores del sistema internacional requiere crear previamente alianzas internas que faciliten la actuación de la región como bloque.

Para que América del Sur logre este tipo de actuación debe consensuar la funcionalidad de los procesos de integración y el rol que estos ocupan en la política exterior de cada uno. UNASUR, como proceso de integración aún en construcción, puede constituirse en un espacio que permita el diálogo y el consenso sobre estos conceptos y a la vez en un foro que facilite la coordinación e integración de políticas regionales.

Considerando estos elementos, la presencia de actores extrarregionales como China e India pone de manifiesto la urgencia de crear ámbitos que permitan a los países sudamericanos abordar los desafíos y oportunidades que estos generan. El consenso es fundamental para evitar la profundi- zación de las asimetrías y la fragmentación regional y para lograr el fortalecimiento de la región como polo de atracción. Reconocemos que la convergencia de los intereses y voluntades de los Estados de América del Sur no son condiciones suficientes para una genuina integración. Coincidimos con la afirmación de que estos procesos pueden concretarse más exitosamente con la presencia de un líder. En el caso de UNASUR, Brasil puede ocupar este lugar si logra respetar la diversidad regional y si tiene la capacidad de coordinar las esferas regional y doméstica (Soares de Lima, 2008: 110). De esta forma debería incentivar a las partes a comprometerse con la integración, las cuales en contrapartida deberían cumplir un rol activo en la definición de los objetivos y métodos de la integración.

La consolidación de la región como bloque a partir de las prácticas de coordinación e integración política dentro del marco de UNASUR, permitirá sentar las bases para una revalorización geoestratégica y económica de la región a nivel internacional. Este proceso llevaría a un mejor posicionamiento regional al momento de negociar, fijar agendas y definir políticas en el concierto de naciones; y por lo tanto fortalecer la posición relativa de América del Sur ante la presencia de actores emergentes de peso como China.

\section{Bibliografía}

Bouzas, Roberto, Pedro da Veiga y Sandra Rios, (2008), "Crisis y perspectivas de la integración en América del Sur», en 
Lagos, Ricardo (comp.) América Latina: ¿Integración o Fragmentación? Buenos Aires, Edhasa, pp. 319-348.

Cesarín, Sergio (2008), «El factor China y los dilemas de gobernabilidad en América Latina y el Caribe», en Res Diplomática, Segunda época, n 3, Buenos Aires, Junio, pp. 6-27.

Cheng, Joseph Y.S. (1999), «China's ASEAN policy in the 1990s: pushing for regional multipolarity", en Contemporary Southeast Asia, 21 (2), August.

Chiaradía, Alfredo (2007), «Integración económica e inserción internacional», en Res Diplomática, Segunda época, n 1, Buenos Aires, Agosto, pp. 93-103.

Cornejo, Romer (2005), "China un nuevo actor en el escenario latinoamericano", en Nueva Sociedad, N. 200, Nov-Dic, pp. 13-24.

Domínguez, Jorge I. (2008), «Las relaciones contemporáneas Estados Unidos - América Latina: entre la ideología y el pragmatismo", en Lagos, Ricardo (comp.) América Latina: ¿Integración o Fragmentación? Buenos Aires, Edhasa, pp. 179-208.

García Moritán, Roberto (2007), «Consideraciones sobre las nuevas fases de la Integración Latinoamericana", en Res Diplomática, Segunda época, No 1 , Buenos Aires, Agosto, pp. 24-33.

Gómez Mera, Laura (2005), «Explaining MERCOSUR's survival: Strategic sources of Argentine-Brazilian convergence», Journal of Latin American Studies, 37, Cambridge University Press, pp. 109-140. Jiang, Shixue (2008), "Una mirada china a las relaciones con América Latina", en Res Diplomática, Segunda época, n 3, Buenos Aires, Junio, pp. 128-147.

Mendoza, María Cecila y Juan Martín Muda (2008), "Argentina, India y el MERCOSUR como puente», en Res Diplomática, Segunda época, n 3, Buenos Aires, Junio, pp. 28-41.

Olivet, María Cecilia (2005), «Unravelling Interregionalism theory: A critical analysis of the new interregional relations between Latin America and East Asia», VI Reunión de la Red de Estudios de América Latina y el Caribe sobre Asia-Pacífico.

Oliva, Carla (2008), «Las posibilidades de un desarrollo armónico", en Res Diplomática, Segunda época, $N^{o} 3$, Buenos Aires, Junio, pp. 42-67.

Peña, Félix (2008), «A integraçao no espaço sul-americano. A Unasul e o Mercosul podem se complementar?", en Nueva Sociedad, Octubre, pp.72-85.

Pinheiro Guimaraes, Samuel (2007), «El mundo multipolar y la integración sudamericana», en Res Diplomática, Segunda época, n 1, Buenos Aires, Agosto, pp. 104-127.

Roett, Riordan (2008), "América Latina y el surgimiento de China en un contexto global», en Lagos, Ricardo (comp.) América Latina: ¿Integración o Fragmentación?, Buenos Aires, Edhasa, pp. 269-289.

Russell, Roberto y Juan Gabriel Tokatlian (2008), «Resistencia y cooperación: opciones estratégicas de América Latina frente a Estados Unidos», en Lagos, Ricardo (comp.), América Latina: ¿Integración o Fragmentación?, Buenos Aires, Edhasa, pp. 209-235.

Sanjuán, Ana María (2008), «América Latina y el bolivarianismo del siglo XXI. Alcances y desafíos de la política venezolana hacia la región», en Lagos, Ricardo (comp.), América Latina: ¿Integración o Fragmentación?, Buenos Aires, Edhasa, pp. 145-176.

Soares de Lima, María Regina (2007), «Brasil como país intermedio: Imprecisión conceptual y dilemas políticos», en Tokatlián, Juan (comp.), India, Brasil y Sudáfrica. El impacto de las nuevas potencias regionales, Buenos Aires, Libros del Zorzal, pp. 169-190.

Soares de Lima, María Regina (2008), «Liderazgo regional en América del Sur: ¿tiene Brasil un papel a jugar?», en Lagos, Ricardo (comp.), América Latina: ¿̇Integración o Fragmentación?, Buenos Aires, Edhasa, pp. 89-114.

Von Grabendorff, Wolf (2008), «La relación América Latina-Unión Europea: entre las percepciones erróneas y las buenas 
Paola Baroni et al. • UNASUR: alternativa de integración frente a desafíos...

intenciones», en Lagos, Ricardo (comp.), América Latina: ¿Integración o Fragmentación? Buenos Aires, Edhasa, pp. 239-268.

Young Jong Choi y Nae Young Lee (2002), «A comparative study of regionalism in East Asia and the Americas", Asian Perspective 26 (3), pp. 169-192.

\section{Referencias Informáticas}

Comisión Eeconómica Para América Latina (2008): Anuario Estadístico, (http:// websie.eclac.cl/anuario_estadistico/anuario_2008/docs/ANUARIO2008.pdf), consultado el 16 de mayo de 2009.

Comisión Estratégica De Reflexión (2006): Un Nuevo Modelo de Integración de América del Sur. Hacia la Unión Sudamericana de Naciones, (http://www.comunidadandina. org/documentos/dec_int/dec_cochabamba_reflexion.htm), consultado el 25 de mayo de 2009

Dirección General De Prensa Presidencial (Venezuela), "Firmados 28 nuevos acuerdos entre Venezuela y China», 25 agosto 2006, (http://www.minci.gob.ve/ noticias-prensa-presidencial/28/10030/ firmados_28_nuevos.html), consultado el 5 de mayo de 2009.

Embajada de la Republica Popular China en India, «Venezuela recognizes China's full market economy status», 24 de diciembre de 2004, (http://www.chinaembassy.org. in/eng/jjmy/t176359.htm), consultado el 15 de mayo de 2009.

Ministerio de Relaciones Exteriores de la República Popular China (2003): China's Stand on South-South Cooperation, (http://www.fmprc.gov.cn/eng/wjdt/wjzc/ t24880.htm), consultado el 15 de mayo de 2009.

Ministerio de Relaciones Exteriores de la República Popular China (2003): China's Views on the Development of Multipolarization, (http:/www.fmprc.gov.cn/eng/ wjdt/wjzc/t24880.htm), consultado el 15 de mayo de 2009.

National Bureau of Statistics of China (2008): China Statistical Yearbook, (http://www. stats.gov.cn/tjsj/ndsj/ 2008/indexeh.htm), consultado el 15 de mayo de 2009.

\section{Artículos periodísticos}

China Daily, «Peru FTA inked in China in quick time», 29 de abril de 2009, (http:// www.asianewsnet.net/news.php? sec= 2\&id=5473), consultado el 15 de mayo de 2009.

China View, "PetroChina sets up joint venture with Venezuela national oil company», 12 de mayo de 2009, (http:// news.xinhuanet.com/english/200905/12/ content_11362659.htm), consultado el 13 de mayo de 2009. 